\title{
A Longitudinal Study of Small Group Interaction in Social Virtual Reality
}

\author{
Fares Moustafa \\ UCL Interaction Centre \\ University College London \\ fmoustafa@gmail.com
}

\author{
Anthony Steed \\ Department of Computer Science \\ University College London \\ A.Steed@ucl.ac.uk
}

\begin{abstract}
Now that high-end consumer phones can support immersive virtual reality, we ask whether social virtual reality is a promising medium for supporting distributed groups of users. We undertook an exploratory in-the-wild study using Samsung Gear VR headsets to see how existing social groups that had become geographically dispersed could use VR for collaborative activities. The study showed a strong propensity for users to feel present and engaged with group members. Users were able to bring group behaviors into the virtual world. To overcome some technical limitations, they had to create novel forms of interaction. Overall, the study found that users experience a range of emotional states in VR that are broadly similar to those that they would experience face-to-face in the same groups. The study highlights the transferability of existing social group dynamics in VR interactions but suggests that more work would need to be done on avatar representations to support some intimate conversations.
\end{abstract}

\section{CCS CONCEPTS}

- Human-centered computing $\rightarrow$ Virtual reality; Computer supported cooperative work;

\section{KEYWORDS}

Virtual reality; social VR; avatar representation; affective states; in-the-wild study

\section{ACM Reference Format:}

Fares Moustafa and Anthony Steed. 2018. A Longitudinal Study of Small Group Interaction in Social Virtual Reality. In VRST 2018: 24th ACM Symposium on Virtual Reality Software and Technology (VRST '18), November 28-December 1, 2018, Tokyo, Japan. ACM, New York, NY, USA, 10 pages. https://doi.org/10.1145/3281505.3281527

\section{INTRODUCTION}

The past couple of years have seen virtual reality reach the consumer market in a significant way. While a lot of attention has been paid to the high-end desktop PC-based systems, devices such as Samsung Gear VR and Google Daydream, which convert a high-end smartphone into a head-mounted display, are now a viable platform for more complex immersive virtual reality environments. Given

\section{(a) $(1)(9$}

This work is licensed under a Creative Commons Attribution-NonCommercial International 4.0 License.

VRST '18, November 28-December 1, 2018, Tokyo, Japan

(C) 2018 Copyright held by the owner/author(s)

ACM ISBN 978-1-4503-6086-9/18/11.

https://doi.org/10.1145/3281505.3281527 that manufacturer's flagship smartphones tend to ship millions of units, such systems are bringing immersive VR to a broad and diverse audience. In particular, as devices become more popular, they may become a viable medium to support collaboration at a distance.

Gaming and social platforms have used non-immersive virtual environments for many years. Certain platforms such as World of Warcraft and Second Life have supported long-lived communities that have been studied from a variety of viewpoints. Certainly, very strong social relationships are formed and maintained on such platforms. However, such platforms have a certain barrier to entry. The form of technology, difficulty of use, or story-based settings have meant that whilst popular, they are not broadly used for social communication. Immersive virtual reality has new affordances over such systems. Whilst social interaction has been studied on immersive systems, this has typically been in a laboratory setting.

In this paper, we describe an exploratory in-the-wild study on the use of immersive virtual reality to support social groups. We are particularly interested in supporting existing groups: thereby we can investigate how groups might migrate to use immersive virtual reality as a collaborative medium as an alternative to voice, picture or text messaging. Participants in our study used social environments on the Samsung Gear VR over an extended period. Through diary studies, quantitative measures and interviews, we explored how they adapt to and use the systems.

The following section will cover the existing literature on how interpersonal relationships are created between people, and the way in which technology has previously supported this type of behaviour. We review current research into the effects avatars have on communication between users in virtual environments, and the way in which relationships can develop between users and avatars. This will provide a basis of contextual understanding that can then be applied to scenarios in VR, as well as act as a basis of comparison between the various technologies. Then, an in-the-wild exploratory study is conducted to gather data on how real users engage with their familiars remotely in VR. We use principles outlined in Grounded Theory $[6,15]$ to analyse the results and discuss how VR can be used as a platform to promote the development of interpersonal relationships.

\section{RELATED WORK}

A great deal of previous research into interpersonal relationships has focused on the importance nonverbal communication plays in human interaction $[1,5,16]$. Steve Duck $[10]$ states that "You cannot utter a word without also simultaneously indicating how you 
feel about the other person" to highlight the significance paralanguage, facial expressions and posture play in the broader context of communication.

Considering the vital role nonverbal communication plays in our everyday interactions, it is critical to consider how virtual environments support nonverbal acts. Collaborative virtual environments (CVEs) have been studied for many years. Such platforms typically represent individual users as an avatar inside a 3D virtual environment where the user can move around the virtual space. The avatar also acts as a mechanism to interact with the world: it may have arms or other means to effect grabbing and moving objects. Thus the avatar serves to represent the user's position, interest and activity [2]. However, even simple avatar representations convey subtle social dynamics.

Fabri et al. [11] highlighted the affect nonverbal communication had on avatar-avatar interactions, noting that facial expressions and posture of avatars triggered emotional responses from players. A study by Moser et al. [20] builds on this and reports that the facial expressions of avatars in VR also have the potential to evoke emotion in users.

Yee et al. [37] conducted an observational study in the online virtual world simulator Second Life (SL) to explore whether social norms surrounding nonverbal communication and interpersonal distance from the real world transferred into virtual contexts. The study established that social behaviors relating to personal distance were present in virtual environments: male-male dyads displayed larger interpersonal distances than female-female dyads, as well as less frequent eye contact. The study also provided evidence that the avatars of the virtual environment would adhere to standard turn taking protocol during conversations as well as noting shifts in user posture during periods of increased levels of intimacy. Further supporting their hypothesis of Equilibrium Theory; that the level of intimacy between dyadic groups is "maintained by compensatory changes in gaze or interpersonal distance" [1, 4, 37].

Similarly, Friedman et al. [12] looked at the social behavior of players in SL when pre-programmed bots would interact with player avatars. The experiment uncovered that when players engaged with the bot they would apply a level of proxemics appropriate to the context. However, contrary to the findings of Yee et al. [37] these did not necessarily mimic real-world behaviors, which the authors claimed was due to a hypothesized lack of presence in virtual environments such as SL.

Presence is the concept that, to some extent, users of immersive virtual reality systems believe that they are in the virtual environment and thus tend to react to the environment in a manner similar to how they would in the real world [32]. Slater's analysis [33] distinguishes 'place presence', which is the illusion of being in a place caused by having the senses immersed in a first person view that is driven by the natural movements of the user, from 'plausibility illusion' which is the impression that what is happening in the virtual environment is happening around the user. A representation of the user as an avatar that the user can see is a key part of both place and plausibility illusion: it both evidences that you are part of the environment, and that events around you are plausible because your body is involved in them. Recent results have shown a fascinating relationship between the virtual body and sense of presence. These include having a type of body ownership over the body [34] and the virtual body having an important role in cognition through supporting gesture [35]. We can thus expect that the combination of immersion and embodiment has an important impact on social behavior.

Collaborative immersive virtual reality systems date back to at least VPL's seminal Reality Built for Two system from the late 1980s. Otto et al. review the use of immersive systems for closely coupled collaboration [21]. They highlight that with immersive systems, users can naturally form different working configurations, where they can observe each other and shared objects. In particular, they highlight that in an immersive system, it is easy to observe where other users are attending, and thus gaze becomes an important resource. Dodds et al. have shown that the users' having animated avatars is an important resource for communication [8]. Our study will focus on a recent generation of Samsung Gear VR that did not come with hand controllers, but we will note examples of how users overcame this.

Previous studies of immersive CVEs have tended to look at laboratory settings over short periods of time. An exception is Steed et al. [36] who studied the way dyadic groups of strangers and friends interacted and collaborated with one another using an immersive virtual reality CAVE. Participants who interacted with friends for several hours quickly establishing methods to perform the group tasks and engaged in small talk unrelated to the task. In contrast, a group of strangers coordinated poorly. Steed et al. speculated that a possible reason for this is an absence of social and interpersonal cues such as authentic facial expression and appearances. Our study is the first that we are aware of that has studied groups of users over an extended period.

Gillath et al. [14] and Prendinger \& Ishizuka [24] have shown that VR can be used as a tool for users to display empathy using virtual characters. Building on this, Riva et al. [25] noted that the degree of presence experienced by users in VR has a direct impact on the emotional states experienced.

Typically, in SL and similar non-immersive CVEs, the user's avatar is on the screen and the user acts through a 3rd person perspective. This breaks the sensorimotor contingencies inherent to immersive virtual reality, and thus makes the relationship to the avatar unique. Schultze and Leahy [31] explore the avatar-self relationship and identify three key components: The Avatar, the Self and The Relationship between the two. This relationship is key in order to attain a sense of virtual presence. They then characterize this into two interrelated categories - (i) telepresence, the feeling that one is actually a part of the world they are experiencing and (ii) social presence, the sense of being in that space with others [4]. The idea of social presence, or co-presence is used widely in related research and is the focus of much of the research in the area (e.g. see [18], [29], and [30]).

Due to their wide availability, and popularity, non-immersive virtual environments such as SL have been studied extensively. Some users have engaged with such systems for years, and thus have formed strong social bonds with other users within the systems. Our study will focus on immersive CVs being introduced to existing social networks. The rationale of this is to understand whether existing social groups transfer successfully, without disadvantaging or discouraging members and thus changing the social group dynamics. 
Because the avatar is usually customizable, an interesting variety of norms and protocols have evolved around avatar representations. Galanxhi and Nah [13] suggest that avatars can be used as masks providing users with a layer of anonymity, creating an emotional distance between themselves and others. Prelinger [23] outlines aspects of the self that people use to identify others such as facial features, gender and physical characteristics. Bessière et al. [3] describes a more 'realistic' approach to avatars, one where the avatar symbolizes an idealistic representation of the communicator. In our study, users will be able to customize avatars and it may be that the immersive situation biases users towards one specific use of avatar representations.

Ducheneaut et al. [9] and Kafai et al. [17] both discuss avatar representation trends from Massively Multiplayer Online Role-Playing Games (MMORPG) and other online virtual environments stating that avatar preferences tend to correspond to more representative portrayals. Mcarthur [19] elaborates on the character creation interface and the experience of creating an avatar, highlighting the importance of identity fidelity, that is, how accurately users are able to represent themselves through their avatars, and attempts to form a unified standard informed by user experience methodologies.

There are several social VR demonstrators for current consumer systems. Our study involved users in Oculus Rooms ${ }^{1}$, AltspaceVR ${ }^{2}$ and vTime ${ }^{3}$.

\section{THE CURRENT STUDY}

\subsection{Design}

Diary entries and semi-structured interviews with groups and individuals were the primary methods of data collection allowing for an in-depth analysis of user experience. In-VR observations also took place with select groups.

A Geneva Emotion Wheel [28] was integrated into the diary study to gauge the intensity of the emotions experienced during sessions, aiding in the affective classification of their experience by plotting them on an Arousal Valence Chart[26].

Claire Petitmengin's [22] method of opening users up to discuss their subjective experience was utilized during interviews to appropriately capture the thought process and affective journey participants felt during their experiences.

\subsection{Participants}

Overall, 17 participants were recruited to take part in the study. This consisted of 9 females and 8 males with an age range of 24-59, and an average age of 30.4 (see Table 1 for more details). Participants were required to create their own groups from existing familiars, and some participants belonged to two groups; for instance, they may have been in one group with their friends and another with siblings. Participants were recruited via social media and were predominantly professionals working in various public and private sectors. None of the participants came from computer science backgrounds, none had any prior experience using or interacting in VR and all were compensated $£ 10$ in Amazon vouchers for taking part taking part in the study.

\footnotetext{
${ }^{1}$ https://www.oculus.com/experiences/go/1101959559889232/

${ }^{2}$ https://altvr.com/

${ }^{3}$ https://vtime.net/
}

Table 1: User group composition in the study

\begin{tabular}{l|lccc} 
& Relation & $\begin{array}{l}\text { Participant Age } \\
\text { number }\end{array}$ & Gender \\
\hline Group A & Friends & P1 & 27 & $\mathrm{f}$ \\
& & $\mathrm{P} 2$ & 30 & $\mathrm{f}$ \\
& & $\mathrm{P} 3$ & 30 & $\mathrm{f}$ \\
\hline Group B & Friends & $\mathrm{P} 4$ & 29 & $\mathrm{~m}$ \\
& & $\mathrm{P} 5$ & 26 & $\mathrm{~m}$ \\
& & $\mathrm{P} 6$ & 28 & $\mathrm{f}$ \\
\hline Group C & Siblings & $\mathrm{P} 7$ & 27 & $\mathrm{~m}$ \\
\hline & & $\mathrm{P} 6$ & - & - \\
\hline Group D & Long & $\mathrm{P} 9$ & 24 & $\mathrm{f}$ \\
\hline & distance & & $\mathrm{m}$ \\
& couple & $\mathrm{P} 10$ & 29 & $\mathrm{f}$ \\
\hline Group E & Siblings & $\mathrm{P} 11$ & 29 & $\mathrm{~m}$ \\
& & $\mathrm{P} 12$ & 24 & $\mathrm{f}$ \\
\hline Group F & Siblings & $\mathrm{P} 2$ & - & - \\
& & $\mathrm{P} 13$ & 27 & $\mathrm{~m}$ \\
\hline Group G & Parent/Child & $\mathrm{P} 7$ & - & - \\
& & $\mathrm{P} 14$ & 59 & $\mathrm{f}$ \\
\hline Group H & Parent/Child & $\mathrm{P} 15$ & 25 & $\mathrm{~m}$ \\
& & $\mathrm{P} 16$ & 50 & $\mathrm{f}$ \\
\hline Group I & Friends & $\mathrm{P} 15$ & - & - \\
& & $\mathrm{P} 17$ & 25 & $\mathrm{~m}$ \\
\hline & & & &
\end{tabular}

\subsection{Materials}

A combination of Samsung Galaxy S6s and S7s were used for the study (16 S7s and $1 \mathrm{~S} 6$ ). Each smart phone was configured with android version 7.0 and the latest version of the Oculus app installed. A Samsung Gear VR SM-R322 was given to each participant to be used in conjunction with the phones. Each participant was also given a dedicated microphone integrated earphone if they didn't have any to use.

\subsection{Procedure}

Participants took part in the study in designated groups of familiars. Once these groups were established the investigator contacted all participants to discuss the study in detail and provide a forum where participants could raise questions, as well as give consent to take part.

At this point, all necessary equipment was given to participants. A set of introductory activities was sent to all participants via email detailing the necessary steps to familiarize users with VR. Diary study templates were made available to all participants by the investigator, participants were required to fill in a diary entry after each session and return the results by the end of the study week.

A total of 8 semi-structured interviews across all 9 groups took place either in a public area or over Skype. The audio from these interviews were recorded using dedicated software after obtaining consent from the participant(s) involved. Interviews took no longer than an hour and were spread over a 4-week period. 
The study was approved by UCLIC's Departmental Ethics Committee, reference UCLIC/1617/008/MSc Steed/Moustafa.

\section{RESULTS}

\subsection{Breakdown of User Activity}

Participants were asked to engage in 2 to 3 social VR sessions a week with their groups for approximately 20-30 minutes at a time. However this was rarely the case: groups frequently opted to have fewer sessions (usually 1 a week, but on occasion, 2) which lasted longer. Over a period of 4 weeks, 33 diary entries were returned with an average time of 49.80 minutes per session (shortest: 20 minutes, longest: 150 minutes). These figures along with anecdotal evidence collected from diary studies and semi- structured interviews highlight the disposition for users to treat social VR experiences similarly to real life outings where groups of people are inclined to meet and carry out some sort of activity, as opposed to using the platform for more casual social interactions similar to telephone catch ups or quick conversations.

P3: "With VR you can do so much more, and experience more, which you can't do in real life or with skype. It's like a different goal that you have. The goal is to hang out, but also to experience this immersive world. If you're communicating with skype then the point is to catch up, not to have a shared experience."

Interestingly, despite being instructed to take part in the study in predesignated groups, participants were quick to divide themselves into sub-groups on occasion where members of a group would coordinate amongst themselves to have their own meet-ups in VR in the absence of other group members. Such behavior demonstrates a similarity to the way in which meet-ups are organized in real life.

Of the 33 sessions that were recorded, 27 of those (82\%) were done using the Oculus Rooms app, 4 sessions using AltspaceVR $(12 \%)$ and 2 using vTime (6\%). Participants were given the freedom to explore the various social VR apps available, however most opted to solely engage in Oculus Rooms due to its tight integration with the Gear VR UI, and the relative ease of which sessions could be created.

Whilst engaging in social VR participants carried out a vast range of activities, this predominantly consisted of:

(1) Watching TV or video clips

(2) Sharing an immersive $360 \mathrm{VR}$ experience

(3) Playing games

(4) Catching up with group members

All participants found that the best use for social VR lies within group engagement and the ability to participate in shared experiences with familiars. It was during these moments of shared activity that users felt most engaged with fellow group members, finding themselves feeling present in the company of others on an intrinsically human level.

P10: "It was really nice to be able to share activities and talk with someone that is far, it actually felt that we were close."

P13: "I feel that (for example) when watching the videos, it was a great way to interact and engage with someone without them physically being there. You definitely feel as if you are there watching the video with the other person in a virtual world and I think that VR is a great way to enjoy other people's company when they can't physically be there with you"

It appears this dynamic was not as present when most users would engage in more intimate one-on-one sessions. The data shows that during moments of intimacy between dyadic groups a lack of dynamic facial expressions and gestures made communication somewhat unrealistic.

P7: "I think in a group its more natural and free flowing. It's harder one on one... maybe that's actually where body language is important. Somehow being one on one with mum is different. I find it's easier in person, there are no awkward gaps in person."

This was the case for all group types except Group D; a couple engaging in a long-distance relationship. Reports from Group D describe that their experiences together in this virtual space always felt intimate regardless of the activity they were engaged in.

P9: "The experience was a refreshing way to interact with P10 from a long distance... Being able to commit to a series of activities together made me feel closer to the person I was talking to."

P10: "...even just chatting to P9 felt intimate, being able to see him and his lips move made me feel closer to him"

One could speculate that the nature of their relationship could have attributed to this feeling of intimacy; that their familiarity with one another transcends the current boundaries of the platform to a point where they still feel genuinely close despite the lack of feedback that usually comes from nonverbal communication $[1,5$, $10,16]$.

\subsection{Affective States in Social VR}

As part of the diary study, participants were required to rank the various relatable affective states throughout each session using a Geneva Emotion Wheel [28]. This provided a much-needed way of categorising the overall tone of the sessions, as well as an opportunity to study any trends and similarities that would arise over time. Affective states were grouped into Positive/Negative and High/Low Arousal states to further segment the range and significance of each response [26].

A breakdown of the data can be seen in Table 2 each affective state is listed on the left under the appropriate valence [26], with a scale ranging from 1-6; 1 being very low (i.e. the recipient could barely relate to the affective state), 6 being very high (i.e. the recipient could highly relate to the affective state).

4.2.1 Positive Valence Readings. As the data shown in Table 2 clearly indicates, there was a significant propensity for users of social VR applications to experience some degree of positive affective states during sessions. $81 \%$ of the total affective states recorded 
Table 2: Summary of report of affective states

\begin{tabular}{|c|c|c|c|c|c|c|c|}
\hline \multirow{2}{*}{$\begin{array}{l}\text { Affective } \\
\text { State }\end{array}$} & \multicolumn{6}{|c|}{ Rating } & \multirow[t]{2}{*}{ Total } \\
\hline & 1 & 2 & 3 & 4 & 5 & 6 & \\
\hline & \multicolumn{6}{|c|}{ Positive - High Arousal } & 128 \\
\hline Pride & 2 & 1 & 3 & 2 & 4 & & 12 \\
\hline Amusement & & & 3 & 14 & 14 & 13 & 44 \\
\hline Joy & & 6 & 8 & 9 & 5 & 6 & 34 \\
\hline \multirow[t]{2}{*}{ Interest } & & 2 & 4 & 7 & 16 & 9 & 38 \\
\hline & \multicolumn{6}{|c|}{ Positive - Low Arousal } & 89 \\
\hline Love & & 1 & 1 & 1 & 1 & 6 & 10 \\
\hline Pleasure & 1 & 4 & 8 & 6 & 7 & 2 & 28 \\
\hline Admiration & & 3 & 2 & & 4 & & 9 \\
\hline Relief & 1 & 1 & 1 & & & & 3 \\
\hline Compassion & & 2 & 1 & 1 & 1 & & 5 \\
\hline \multirow[t]{2}{*}{ Contentment } & & 5 & 6 & 6 & 11 & 6 & 34 \\
\hline & \multicolumn{6}{|c|}{ Negative - High Arousal } & 19 \\
\hline Fear & 1 & & 1 & 2 & & & 4 \\
\hline Anger & 1 & 4 & 2 & 1 & & 1 & 9 \\
\hline Hate & & 1 & & & & & 1 \\
\hline Contempt & 1 & 1 & 1 & & & & 3 \\
\hline \multirow[t]{2}{*}{ Disgust } & 1 & & 1 & & & & 2 \\
\hline & \multicolumn{6}{|c|}{ Negative - Low Arousal } & 32 \\
\hline $\begin{array}{l}\text { Disappoint- } \\
\text { ment }\end{array}$ & 1 & 5 & 6 & 6 & 4 & 1 & 23 \\
\hline Sadness & 1 & 1 & 2 & 1 & & & 5 \\
\hline Shame & & & & & & & 0 \\
\hline Guilt & & & 1 & & & & 1 \\
\hline Regret & & & 2 & 1 & & & 3 \\
\hline
\end{tabular}

corresponded to feelings belonging to a positive valence. Participants from all groups stated that this affinity to positive affective states was a direct result of the level to which they felt engaged with their group members and that how interacting in this platform contributed to their feeling of social-presence.

P10: "It was much better than I had expected, because it was nice to share activities alongside just simply talking."

The most prominent affective states participants identified with were Amusement closely followed by Interest, Joy and Contentment. Participants felt that interacting with group members in VR provided an additional dimension to more traditional forms of communication such as the telephone or texting, as well as more modern forms such as Skype or Facetime.

P5: "I definitely found it more natural and personal than a phone call. Just because you have something to look at and they're looking back at you. Even if you can't do much with your avatar, at least you have that connection, looking at each other. I find skype video and stuff just awkward to use."

This elevated level of presence allowed for a more naturalistic form of communication between groups, catering to the sense of

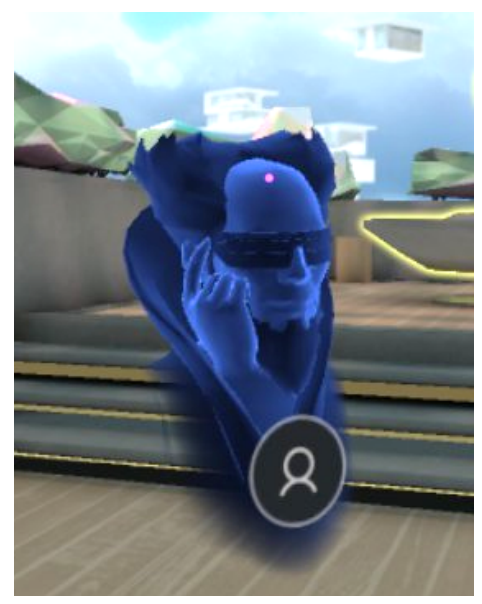

Figure 1: A user making a 'rude' gesture.

mischief and adventure which often accompanies a social gathering of friends. This was something that was very evident during observations of groups and it was a recurring theme in diary entries.

The researcher witnessed such an occasion during an observational study of Group B. The camaraderie and jovial nature of their interactions was evident from the start. They approached the experience in a light-hearted manner and were quick to poke fun at one another the way close friends do. Based on their feedback, the way in which they interacted with one another was directly comparable to how they would do so outside of VR.

During this observation, the researcher noted that participants were, at times, raising their hands to press the 'enter' button on the track pad located on their headset - doing so would display a representation of the users' hand in VR. When asked about this during the post observation interview with the group, one of the participants elaborated, stating that the group had developed a way of signaling a crude gesture to each other in the absence of the ability to raise their middle fingers in their VR sessions. A screen shot of the gesture can be seen in Figure 1.

Whilst there are controllers to track hand movements in VR, these were not included as part of this experiment. Regardless, groups were still able to adapt to their environment and use the capabilities of the system to a suit their needs. In a similar scenario, Group E (a dyadic group of siblings) began to wave goodbye to each other using the motion from their heads.

P11: "at the end we tried to say goodbye by shaking our heads and I had fun"

Multiple groups emphasized the power one's voice had in conveying emotion and intent. In the absence of other nonverbal gestures participants began to note how they were able to decode signals that would traditionally be accompanied by facial or hand gestures just by listening to the tone of their group members' voice.

P2: "You do pick up on all the nuances of people's voice so it feels like you're actually talking to them. I can tell when they're being sarcastic and stuff." 
4.2.2 Negative Valence Readings. Whilst at first glance it may seem that users relating to affective states which correspond to negative valences may be indicative of a negative experience, this is not necessarily the case. It is important to distinguish between experiences that were genuinely negative due to the hardware or technological platform and those that occurred naturally from interpersonal relationships, such as annoyance at an individual for something they said or did during a session, or the sadness one feels when reminded of the geographical distance between them and their familiars.

Technological Issues. This category focuses on the challenges experienced specifically with the current technology and can be further subcategorized into a) issues experienced with the physical hardware (i.e. the headset and the phone) and b) issues experienced with VR design and social VR apps.

Hardware Issues. All participants reported difficulty with hardware on some level at various intervals of the study. This was most prominent for P11 who due to initial resourcing issues was given an older phone model, namely a Samsung Galaxy S6. This participant was the only person using this phone model and frequently had issues with the phone overheating and applications crashing. These issues were not limited to the S6, however they were the most prominent and the most severe for P11 and consequently reflected this in his diary entries.

All 17 participants (100\%) reported audio difficulties to some degree, most prominent of which were echoes and delays in speech This disrupted the flow of conversation and skewed the turn-taking protocol making it harder for users to determine when a person was about to speak.

A total of 8 participants (47\%) mentioned the heat and weight of the device acting as a barrier during sessions, whilst not enough to deter them from sessions it did have occasional implication on their immersion.

Challenges Related to VR. This category reflects on the challenges participants experienced whilst engaged in VR. The most prominent issue that came out of diary entries was the inability to move around or behave "naturally". 12 participants (71\%) expressed their desire to be able to move their hands, to move within the shared space more freely and be able to express facial expressions. Even amongst those that adapted their way of communicating found themselves wanting to be more expressive. Group E noted the cultural importance of hand gestures during social interactions and found the lack of these gestures impacted their initial experiences.

Group C were most affected by the absence of facial expressions; to the point where it made some sessions uncomfortable. They dealt with this by averting each other's gaze and focusing on items in the room.

Interestingly P6 did not have the same experience when interacting with Group B. She found that the intimacy of a one-to-one interaction with her sister required the facial and bodily expressiveness she had come to expect and any deviation from that would impede on her sense of immersion and presence. Whereas when interacting with her friends in a jovial manner the significance of such expressiveness wasn't as important.

The second most prominent issue raised by 8 participants (47\%) was the lack of free and compelling applications in VR.



Figure 2: An example of a user disconnecting from the room.

Finally, 3 participants (18\%) found the way in which Oculus Rooms deals with users dropping out of sessions to be disturbing: avatars would begin to move erratically then eventually slow to a halt, turning grey and eventually disappear. See Figure 2. Participants who experienced the drop out related to affective states such as Fear and Sadness.

Affective Responses. On the other side of the spectrum, a portion of the negative affective states recorded corresponds to emotions that arise organically through interaction, and not necessarily a barrier to the development of interpersonal relationships.

P4 \& P7 recall their first session together with the other members of Group B, a close group of friends who met during their undergraduate years at university. The group no longer see each other as often as they used to. P7 moved abroad 2 years ago after accepting a job offer while the other members of the group have scattered around different locations in London. It wasn't long before P4 \& P7 began to bicker during their first encounter, which quickly developed into a desire for boisterous physical confrontation, reminiscent of their behavior when they were roommates.

P7: "... he's quite haha, he's quite bossy. And he'd already been on it [Oculus Rooms] before so he was like "do this, do this, do this". Obviously like, I started getting annoyed at him, and I just wanted to jump on him, but I couldn't do that so I tried to headbutt him instead... But that's just us, we just get very frustrated at each other haha. That's when it felt like we were actually there together."

P4: "Me and P7 were joking around like we normally do, throwing jokes and insults at each other. He said his avatar could come over and headbutt me, and you could see his avatars head moving back and forth. We were joking like we would in real life. Me getting annoyed because he wouldn't stop going on about headbutting 
me. That felt real, the annoyance level, and the joking level ha."

While P4's diary entry for that session rated Anger at a 4, it's clear that the core of that affective state is routed in a deep interpersonal bond between the two. Both found that they were able to express true nature of their relationship in VR despite being situated in different parts of the world.

P7 goes on to describe how the platform helped bring to light feelings of homesickness he was previously unaware of, as well as using the same platform to alleviate some of the symptoms. Since moving abroad he has had little physical contact with friends or family from the U.K. His initial diary entries reflected emotions of Sadness, Love, Contentment and Amusement (ranking those at 4, 5 , 6,6 , respectively). This highlights the impact being in a room with his friends had on him.

P7: "I think it's... a little bittersweet. Because I'm so far from everyone it's not the same as actually being there... [but] it felt like we were all in the room together, it was nice"

P7: "I was thinking about it - it's homesickness, that's what I'm feeling now. Maybe I'm feeling it retrospectively, but I think definitely that these experiences; coming back home and then flying back here are always going to be a downer. Maybe that's what VR is to someone that's homesick, [a way to experience being with the ones you miss] ... that stuff is kind of hard to talk about."

Throughout the study P7's feelings of Sadness appeared to fade entirely (ranking at a 0 in later entries), however his sense of Love, Contentment and Amusement remained relatively high (around 3-6).

During a session in AltspaceVR P11 notes the discomfort he felt when other users would get too close to him

P11: "I felt discomfort when they pushed a stick with food into my virtual mouth (I had the feeling of a stick being pushed into my throat) ... also other avatars were getting very close to me, "almost touching", and I felt very uncomfortable that they were not respecting my space (even I know it was just virtual space)"

During that session, he related to feelings of Disgust, Hatred and Anger as a stranger came up to him and attempted to "force feed" him. Unable to stop them from doing so, he felt his only option was to $\log$ out.

\subsection{Avatar Portrayals - Findings}

A secondary aim of this study was to determine how varying avatar portrayals in VR affects the avatar-self relationship[31], as well as the perception of others when interacting with the avatar.

9 participants (53\%) created avatars that were representative of their current selves choosing to either create avatars that physically resembled themselves or an aspect of their personality.

A total of 5 participants (29\%) created avatars that were entirely fictionalized. Of the 5 participants, $1(6 \%)$ used the 'randomize'

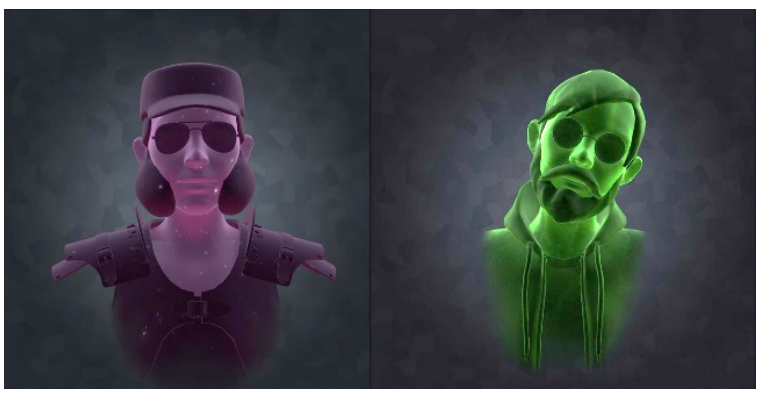

Figure 3: One user's avatar before and after a discussion with their partner.

feature included in the Oculus Rooms Avatar Editor while the others opted to customize their own

One $(6 \%)$ participant experimented with both representative and fictional avatar portrayals, whilst $2(12 \%)$ participants didn't customize their avatars at all and used the default avatar assigned to them in Oculus Rooms.

4.3.1 Changes to Avatars. During the study, 11 participants (65\%) would make some sort of alteration to their avatar, while $4(24 \%)$ participants kept their initial selection. The reasons behind most of the alterations were to experiment with the various representations available. However, there were several instances where participants felt obligated to change their avatar or forced to do so by their familiars.

P6 stated that during her first session with Group B she felt that her avatar was unremarkable in comparison to the avatars of her familiars and as such felt obliged to change it.

P6: "When I first set it up [her avatar] I tried to make it as close to myself as possible. And when we went into the actual room to have a chat I realized everyone had gone really funky with theirs, so I thought 'oh maybe I should play around with it a bit as well'".

P9 felt 'forced' to change his initial avatar selection once his partner associated his virtual representation with the Cuban revolutionary Fidel Castro. Whilst this was a humorous topic for the two, P10 found the representation made her too uncomfortable to communicate naturally with $\mathrm{P}$, finding the physical similarities to Castro very distracting (see Figure 3).

P10: "I didn't feel comfortable hearing his voice come out of the avatars head; with that image ... maybe it's because he was a historical figure, but I couldn't handle it"

P9: "Yeah, so I changed my avatar and went for a more normal look, a hipster look ... you were definitely more endearing, more open when I changed my avatar"

When asked about how changes in avatar representation affected group members, participants on the whole agreed that smaller changes would have little impact to the way they perceive others. They relued heavily on paralanguage to distinguish the identity of users over visual representations. 
Group C discussed how their avatar representations made it more difficult for them to engage with one another. This was comparable to the difficulties they had with the lack of nonverbal communication.

P6: "... It was kind of uncomfortable. It looked nothing like her, at all! I don't know why it was so different [when compared to her interactions with avatars from Group B], but it was uncomfortable."

P6's response when asked to identify what specifically made her feel uncomfortable:

P6: "the hair.... I know that's strange. She picked an avatar with really curly hair... she looked very 70s, she looked cool, it was a cool avatar. But it did not suit her personality as far as I would say. I think when I socialize with my sister ... I'm so used to seeing her facial expressions and quirks."

\subsection{Data Analysis - Recurring Themes}

Theme analysis was based on grounded theory $[6,15]$. All data from diary entries, interview transcripts and observational studies were gathered and split into the following categories concerning affective themes: those that correspond to social experience, those that corresponded to avatar representation, and those that corresponded to hardware. From this, we were able to classify the following as recurring themes of the study.

Users tend to mimic real-world behavior. All participants reported a heightened sense of presence and immersion whilst engaging in VR.

Affective experiences in VR are comparable to affective experiences in the real world. Most participants that were interviewed about their experiences closely related their affective responses to stimuli in VR similarly to experiences in real life.

Social VR is better suited for group interactions over one-on-one interaction. In its current form, social VR apps appear to cater more to the needs of group dynamics over intimate conversation between two individuals.

Limited mobility. Participants shared a desire to be able to take off HMDs more easily whilst still engaging with group members in sessions.

Difficulty in interpreting conversation protocol without nonverbal cues. Users found it difficult to determine when another person was about to speak or wanted to speak. Whilst the social VR applications include animated mouths during times of speech, there is no cue to let users know when a group member is about to speak.

Avatars tend to be representative of users. Avatar representations in a social VR context were predominantly representative of the individual user, whether it was a lifelike portrayal or representative of their personality.

\section{DISCUSSION}

The findings from our exploratory study provide evidence to suggest that users engaged in social VR have an inclination to experience stimuli in VR similarly to the way they would in real life. Interaction with their familiars in VR was directly comparable to time spent together in the real world and evoked genuine affective responses. This discovery builds on the study of Friedman et al. [12], who reported discrepancies in the way users behaved in virtual environments due to a perceived lack of presence. However, the results of this study show a similarity between behavior in the real world and behaviors in VR suggesting that the level of presence and immersion users feel whilst engaged in VR contribute to a more lifelike experience.

Furthermore, there is evidence to support findings from Yee et al. [37] and Friedman et al. [12] that users assign a degree of spatial awareness to their surroundings in VR in the same way they do in the real world. Invading a user's space in VR is a potentially distressing situation akin to harassment. The effects of such behavior may be amplified in immersive systems due to the perception of space and user surroundings.

Whilst these are significant findings contributing to the effectiveness of developing interpersonal relationships in VR, several barriers prevented users from experiencing the full potential of the technology. Most notably was the absence of some forms of nonverbal communication. The inability to supplement interactions with lifelike facial expressions or hand gestures made it difficult to interpret certain social cues. This was particularly evident during more intimate one-on-one interactions. However, there was evidence to suggest that users acclimatized to the absence of nonverbal communication over time.

Group dynamics remained largely unchanged between friends, couples and siblings. This confirms that familiars experiencing emotions inter-subjectively is, in and of itself, a stimulus to deepen interpersonal bonds[7]. This is supported the overwhelming inclination to relate to emotional states which correspond to positive valences.

\subsection{Gesture and Embodiment}

The fact that users were able to naturally express themselves in VR and decipher intent through vocal tones, is indicative to theories stated by Friedman et al. [12] and Sanchez-Vives and Slater [27] that an increased feeling of presence evokes a more humanistic interaction between users. Examples of both telepresence and social presence were evident in interactions between users. Users reported feeling comfortable with their personal representation and feeling present in the virtual space with their group members. This coupling of presence seems to be one of the key elements which allows users to interact naturally with one another in VR.

There was also evidence to support that users were able to relate to their environment regardless of the fidelity, to the point where they attempted to engage with virtual objects that were in front of them. Given that current social VR applications have a stylized aesthetic suggests that virtual environments do not have to mimic photorealistic objects for users to feel immersed in their environment. It also suggestions that the role of virtual space is perhaps more impactful than in non-immersive virtual environments. 


\subsection{Avatar Representations}

The data collected on avatar representation was consistent with the theory put forward by Bessière et al. [3] that suggests that users tend to opt for more lifelike or representative avatar portrayals. This was an important element in establishing the avatar-self relationship [31] and telepresence [4] within the environment. A predisposition to representative avatars in social VR may exist due to the nature of the interaction. Users were almost always engaging with people they were familiar with and thus, potentially more open to represent themselves in a way that closely resembles their physical selves.

\subsection{Limitations}

Although novel in its focus and scope, the study was based on a relatively small set of users. The users were predominantly based in the UK, and thus while this is a large market for these systems, in other cultures, user responses might be quite different. It might also be different if groups are made up of quite distinct personalities and cultures. A larger scale study, crossing different continents would be a logistical challenge, but would present interesting new questions, especially as it could focus on groups where members might only rarely be in physical contact.

It is also worth noting that all participants were given a secondary phone to use specifically for this study. It is unclear how this may have affected the results, particularly in the organization of meet-ups. However, we speculate that the overall experience would have been altered if participants were using their personal phones as the primary medium to engage in VR. With the trend now in the market to supporting secondary fully-integrated VR devices that are roughly equivalent in power to Gear VR and with similar affordances (e.g. Oculus Go or Vive Focus) we might expect that consumers might have different reactions depending on whether they are using their primary communication device or a secondary one. At the very least it might mean that they need to $\log$ in a second time to the secondary device, and this might then mean that they have fewer options for collaboration. Alternatively, they might use both devices with the primary device as a backup coordination mechanism, or to provide continuous text chat. This whole issue of device adoption deserves further study.

\section{CONCLUSIONS}

The study consisted of an exploratory in-the-wild study where participants were required to engage with group members in social VR applications. We gauged participants' affective responses to the stimuli experienced during sessions. This was supplemented with diary studies, semi structured interviews and observational studies of user interactions in VR. The study was purposefully set up in contrast to the more common lab-based studies. It thus solicited, over a much longer engagement with the media, the key issues of practical use and engagement that consumers went through with social VR. Compared to previous studies it also targeted preexisting groups that are transferring to social VR to support their communication.

The evidence gathered as part of this investigation demonstrates a strong propensity for users to experience emotional states in VR similarly to how they are experienced in real life. It strongly suggests that users experienced co-presence or telepresence with their group members. Additionally, the study highlights the transferability of existing social group dynamics in to VR interactions. The study brought to light the way in which users of immersive social VR applications portray themselves as avatars. It revealed that users were inclined towards lifelike or characteristic representations of themselves. While many of these issues are previously discussed in the literature, encountering them as the lived experience of participants in an in-the-wild study has allowed us to re-raise specific issues of representations and add new dimensions to the criteria we might expect for broad adoption of the technology.

\section{ACKNOWLEDGMENTS}

We would like to acknowledge Dr Raz Schwartz from Facebook for his support of the project, including the loan of the Oculus equipment. We also acknowledge the Context Aware Network Architectures for Sending Multiple Senses (CASMS) project, funded by UK's EPSRC (EP/P004040/1).

\section{REFERENCES}

[1] Michael Argyle. 1988. Bodily Communication 2nd Ed. Methuen, London.

[2] Steve Benford, John Bowers, Lennart E. Fahlén, Chris Greenhalgh, and Dave Snowdon. 1995. User Embodiment in Collaborative Virtual Environments. In Proceedings of the SIGCHI Conference on Human Factors in Computing Systems (CHI '95). ACM Press/Addison-Wesley Publishing Co., New York, NY, USA, 242249. https://doi.org/10.1145/223904.223935

[3] Katherine Bessière, A. Fleming Seay, and Sara Kiesler. 2007. The Ideal Elf: Identity Exploration in World of Warcraft. CyberPsychology \& Behavior 10, 4 (Aug. 2007), 530-535. https://doi.org/10.1089/cpb.2007.9994

[4] Frank Biocca, Chad Harms, and Judee K. Burgoon. 2003. Toward a More Robust Theory and Measure of Social Presence: Review and Suggested Criteria. Presence: Teleoperators and Virtual Environments 12, 5 (Oct. 2003), 456-480. https://doi. org/10.1162/105474603322761270

[5] Judee K. Burgoon and Joseph B. Walther. 1990. Nonverbal Expectancies and the Evaluative Consequences of Violations. Human Communication Research 17, 2 (Dec. 1990), 232-265. https://doi.org/10.1111/j.1468-2958.1990.tb00232.x

[6] Juliet M. Corbin and Anselm Strauss. 1990. Grounded theory research: Procedures, canons, and evaluative criteria. Qualitative Sociology 13, 1 (March 1990), 3-21. https://doi.org/10.1007/BF00988593

[7] Anne M. Cronin. 2014. Between friends: Making emotions intersubjectively. Emotion, Space and Society 10 (Feb. 2014), 71-78. https://doi.org/10.1016/j.emospa. 2013.03.008

[8] Trevor J. Dodds, Betty J. Mohler, and Heinrich H. BÃČ Âijlthoff. 2011. Talk to the Virtual Hands: Self-Animated Avatars Improve Communication in HeadMounted Display Virtual Environments. PLOS ONE 6, 10 (Oct. 2011), e25759. https://doi.org/10.1371/journal.pone.0025759

[9] Nicolas Ducheneaut, Ming-Hui Wen, Nicholas Yee, and Greg Wadley. 2009. Body and Mind: A Study of Avatar Personalization in Three Virtual Worlds. In Proceedings of the SIGCHI Conference on Human Factors in Computing Systems (CHI '09). ACM, New York, NY, USA, 1151-1160. https://doi.org/10.1145/1518701.1518877

[10] Steve Duck. 2007. Human Relationships (4th edition ed.). SAGE Publications Ltd, Los Angeles.

[11] Marc Fabri, David J. Moore, and Dave J. Hobbs. 1999. The Emotional Avatar: Non-verbal Communication Between Inhabitants of Collaborative Virtual Environments. In Gesture-Based Communication in Human-Computer Interaction (Lecture Notes in Computer Science), Annelies Braffort, Rachid Gherbi, Sylvie Gibet, Daniel Teil, and James Richardson (Eds.). Springer Berlin Heidelberg, 269-273.

[12] Doron Friedman, Anthony Steed, and Mel Slater. 2007. Spatial Social Behavior in Second Life. In Intelligent Virtual Agents (Lecture Notes in Computer Science), Catherine Pelachaud, Jean-Claude Martin, Elisabeth André, Gérard Chollet, Kostas Karpouzis, and Danielle Pelé (Eds.). Springer Berlin Heidelberg, 252-263.

[13] Holtjona Galanxhi and Fiona Fui-Hoon Nah. 2007. Deception in cyberspace: A comparison of text-only vs. avatar-supported medium. International fournal of Human-Computer Studies 65, 9 (Sept. 2007), 770-783. https://doi.org/10.1016/j. ijhcs.2007.04.005

[14] Omri Gillath, Cade McCall, Phillip R. Shaver, and Jim Blascovich. 2008. What Can Virtual Reality Teach Us About Prosocial Tendencies in Real and Virtual Environments? Media Psychology 11, 2 (June 2008), 259-282. https://doi.org/10. $1080 / 15213260801906489$ 
[15] Barney Glaser and Anselm Strauss. 1967. The Discovery of Grounded Theory: Strategies for Qualitative Research. Aldine.

[16] Laura K. Guerrero and Kory Floyd. 2005. Nonverbal Communication in Close Relationships (1 edition ed.). Routledge, Mahwah, N.J.

[17] Yasmin B. Kafai, Deborah A. Fields, and Melissa S. Cook. 2010. Your Second Selves: Player-Designed Avatars. Games and Culture 5, 1 (Jan. 2010), 23-42. https://doi.org/10.1177/1555412009351260

[18] Matthew Lombard, Frank Biocca, Jonathan Freeman, Wijnand IJsselsteijn, and Rachel J. Schaevitz (Eds.). 2015. Immersed in Media: Telepresence Theory, Measurement \& Technology (2015 edition ed.). Springer, Cham.

[19] Victoria McArthur. 2017. The UX of Avatar Customization. In Proceedings of the 2017 CHI Conference on Human Factors in Computing Systems (CHI '17). ACM, New York, NY, USA, 5029-5033. https://doi.org/10.1145/3025453.3026020

[20] Ewald Moser, Birgit Derntl, Simon Robinson, Bernhard Fink, Ruben C. Gur, and Karl Grammer. 2007. Amygdala activation at 3T in response to human and avatar facial expressions of emotions. Fournal of Neuroscience Methods 161, 1 (March 2007), 126-133. https://doi.org/10.1016/j.jneumeth.2006.10.016

[21] Oliver Otto, Dave Roberts, and Robin Wolff. 2006. A Review on Effective Closelycoupled Collaboration Using Immersive CVE's. In Proceedings of the 2006 ACM International Conference on Virtual Reality Continuum and Its Applications (VRCIA '06). ACM, New York, NY, USA, 145-154. https://doi.org/10.1145/1128923.1128947

[22] Claire Petitmengin. 2006. Describing one's subjective experience in the second person: An interview method for the science of consciousness. Phenomenology and the Cognitive Sciences 5, 3 (Dec. 2006), 229-269. https://doi.org/10.1007/ s11097-006-9022-2

[23] Ernst Prelinger. 1959. Extension and Structure of the Self. The fournal of Psychology 47, 1 (Jan. 1959), 13-23. https://doi.org/10.1080/00223980.1959.9916303

[24] Helmut Prendinger and Mitsuru Ishizuka. 2005. The Empathic Companion: A Character-Based Interface That Addresses Users' Affective States. Applied Artificial Intelligence 19, 3-4 (March 2005), 267-285. https://doi.org/10.1080/ 08839510590910174

[25] Giuseppe Riva, Fabrizia Mantovani, Claret Samantha Capideville, Alessandra Preziosa, Francesca Morganti, Daniela Villani, Andrea Gaggioli, Cristina Botella, and Mariano Alca ̃̃ČÂśiz. 2007. Affective Interactions Using Virtual Reality: The Link between Presence and Emotions. CyberPsychology \& Behavior 10, 1 (Feb. 2007), 45-56. https://doi.org/10.1089/cpb.2006.9993

[26] J. A. Russell and L. F. Barrett. 1999. Core affect, prototypical emotional episodes, and other things called emotion: dissecting the elephant. fournal of Personality and Social Psychology 76, 5 (May 1999), 805-819.

[27] Maria V. Sanchez-Vives and Mel Slater. 2005. From presence to consciousnes through virtual reality. Nature Reviews. Neuroscience 6, 4 (April 2005), 332-339. https://doi.org/10.1038/nrn1651

[28] Klaus R. Scherer. 2005. What are emotions? And how can they be measured? Social Science Information 44, 4 (Dec. 2005), 695-729. https://doi.org/10.1177/ 0539018405058216

[29] Ralph Schroeder. 2010. Being There Together: Social Interaction in Shared Virtual Environments. Oxford University Press.

[30] Ralph Schroeder. 2012. The Social Life of Avatars: Presence and Interaction in Shared Virtual Environments. Springer Science \& Business Media.

[31] Ulrike Schultze and Matthew Leahy. 2009. The Avatar-Self Relationship: Enacting Presence in Second Life. ICIS 2009 Proceedings (Jan. 2009). https://aisel.aisnet. org/icis2009/12

[32] Thomas B. Sheridan. 1992. Musings on Telepresence and Virtual Presence. Presence: Teleoperators and Virtual Environments 1, 1 (Jan. 1992), 120-126. https: //doi.org/10.1162/pres.1992.1.1.120

[33] Mel Slater. 2009. Place illusion and plausibility can lead to realistic behaviour in immersive virtual environments. Philosophical Transactions of the Royal Society B: Biological Sciences 364, 1535 (Dec. 2009), 3549-3557. https://doi.org/10.1098/ rstb.2009.0138

[34] Mel Slater, Daniel Pérez Marcos, Henrik Ehrsson, and Maria V. Sanchez-Vives. 2008. Towards a digital body: the virtual arm illusion. Frontiers in Human Neuroscience 2 (2008). https://doi.org/10.3389/neuro.09.006.2008

[35] Anthony Steed, Ye Pan, Fiona Zisch, and William Steptoe. 2016. The impact of a self-avatar on cognitive load in immersive virtual reality. In 2016 IEEE Virtual Reality (VR). IEEE, Greenville, SC, USA, 67-76. https://doi.org/10.1109/VR.2016. 7504689

[36] Anthony Steed, Maria Spante, Ilona Heldal, Ann-Sofie Axelsson, and Ralph Schroeder. 2003. Strangers and Friends in Caves: An Exploratory Study of Collaboration in Networked IPT Systems for Extended Periods of Time. In Proceedings of the 2003 Symposium on Interactive 3D Graphics (I3D '03). ACM, New York, NY, USA, 51-54. https://doi.org/10.1145/641480.641492

[37] Nick Yee, Jeremy N. Bailenson, Mark Urbanek, Francis Chang, and Dan Merget 2007. The Unbearable Likeness of Being Digital: The Persistence of Nonverbal Social Norms in Online Virtual Environments. CyberPsychology \& Behavior 10, 1 (Feb. 2007), 115-121. https://doi.org/10.1089/cpb.2006.9984 